\title{
Memory-Dependent Forecasting of COVID-19: The Flexibility of Extrapolated Kernel Least Mean Square Algorithm
}

This paper was downloaded from TechRxiv (https://www.techrxiv.org).

\section{LICENSE}

CC BY-NC-SA 4.0

SUBMISSION DATE / POSTED DATE

22-02-2021 / 01-03-2021

\section{CITATION}

Ahmad, Noor; Mohd, Mohd Hafiz (2021): Memory-Dependent Forecasting of COVID-19: The Flexibility of Extrapolated Kernel Least Mean Square Algorithm. TechRxiv. Preprint.

https://doi.org/10.36227/techrxiv.14077361.v1

$\mathrm{DOI}$

10.36227/techrxiv.14077361.v1 


\title{
Memory-Dependent Forecasting of COVID-19: The Flexibility of Extrapolated Kernel Least Mean Square Algorithm
}

\author{
Noor Atinah Ahmad and Mohd Hafiz Mohd
}

\begin{abstract}
The extrapolated kernel least mean square algorithm (extrap-KLMS) with memory is proposed for the forecasting of future trends of COVID-19. The extrap-KLMS is derived in the framework of data-driven modelling that attempts to describe the dynamics of infectious disease by reconstructing the phasespace of the state variables in a reproducing kernel Hilbert space (RKHS). Short-time forecasting is enabled via an extrapolation of the KLMS trained model using a forward euler step, along the direction of a memory-dependent gradient estimate. A userdefined memory averaging window allows users to incorporate prior knowledge of the history of the pandemic into the gradient estimate thus providing a spectrum of scenario-based estimates of futures trends. The performance of the extrap-KLMS method is validated using data set for Malaysia, Saudi Arabia and Italy in which we highlight the flexibility of the method in capturing persistent trends of the pandemic. A situational analysis of the Malaysian third wave further demonstrate the capabilities of our method
\end{abstract}

Index Terms-COVID-19, data-driven modelling, kernel least mean square, machine learning, short-term forecasting.

Note to Practitioners-The method presented in this paper provides event-related and scenario-based forecasting of COVID-19. Our intention is to fill the gap between mechanistic modelling that relies on physical theories and qualitative nature of solutions, and, data-dependent approaches offered by most statistical methods. While extrap-KLMS combines the advantages of mechanistic and statistical modelling, it also inherits the limitations. Nevertheless, our results put forward convincing evidence that under certain circumstances, the advantages outweigh the limitations. The application of extrap-KLMS method is not limited to the forecasting of epidemic time series; it may also be extended to other timeseries forecasting problems and adaptive learning process.

\section{INTRODUCTION}

$\mathbf{T}$ HE end of 2019 witnessed the wide spread of the new coronavirus (COVID-19) in China, infecting a large number of people. Since then, COVID-19 has developed into a global pandemic. A year on, the pandemic is still raging throughout the world and has led to devastating impact on lives and livelihood with millions of people are at risk of falling into extreme poverty. The UN's Framework for the Immediate Socio-Economic Response to the COVID 19 Crisis warns that "The COVID-19 pandemic is far more than a health crisis: it

This work was supported by the Ministry of Higher Education Malaysia under the FRGS grant (Acc no: 203.PMATHS.6711942)

N. A. Ahmad is with the School of Mathematical Sciences, Universiti Sains Malaysia, 11800, Penang MALAYSIA e-mail: nooratinah@usm.my.

M. H. Mohd is with the School of Mathematical Sciences, Universiti Sains Malaysia, 11800, Penang MALAYSIA.

Manuscript received April 19, 2005; revised August 26, 2015. is affecting societies and economies at their core" [1]. Urgent socio-economic and development responses are much needed in this trying time to stem the impact of the pandemic.

The ability to predict the future of epidemics and pandemics is important to help gain better understanding of the current situation. Many policymakers have relied on mathematical models to guide timely, well-informed responses to the pandemic. Although there are a lot of debate on how much faith one should put on the validity of epidemic forecasting and mathematical models [2]-[4], it is inevitable that mathematical models shall continue to be one of the main tools to provide insights and possible solutions. Conventional epidemiological models such as the family of susceptible-infected-recovered (SIR) compartmental models [5]-[8] are among the popular mathematical models used for prediction, mainly due to their sound theoretical basis and a history of useful applications. Epidemiological models are driven by prior assumptions which are translated into a set of assumed parameterized mathematical equations. However, it has been reported that COVID-19 can behave in unexpected ways; for example, asymptomatic cases that can be infectious agents for several weeks [9], [10], there are also reports of different mutations of the virus in certain countries [11]-[13]. These challenges lead to the possibility of unknown dynamics and can limit the ability of conventional models in capturing certain intrinsic trends of the spread.

The baseline solutions to perform prediction tasks are the statistical methods, mostly employing some improved versions of regressive models [20], [21]. Data-driven regression combined with machine learning is becoming vital tools in the prediction of COVID-19 pandemic [14]-[16]. Unlike mechanistic epidemiological models, statistics and machine learning models do not normally include any disease transmission mechanism, thus their forecasts are only reliable within a short time window. Their imposed analytic models can also be restrictive especially when dealing with nonstationarities and nonlinearities of data. Notwithstanding its many potentials, there has been concerns on the sensitivity of this class of methods to data quality [17], [18] and possibilities of overfitting [19]. As reported in [17], the time series of recovered individuals that are released officially are heavily unreliable, an the primary cause resides in the marked non-stationary character of the testing activity. They further demonstrate that overlooking these effects inevitably leads to fundamentally wrong predictions. Another widely used data-driven approaches are the Neural Networks (NN) family of methods [22], [23], 
employing one or more layers of nonlinear units to predict outputs. Nonetheless, NN algorithms tend to demand long training time and may get stuck in local minima. Yet another class of nonlinear machine learning techniques is the class of kernel methods [24], [25]. The main idea behind kernel methods for dynamical system is to reconstruct the phase space in a higher-dimensional spaces (i.e. the so-called reproducing kernel Hilbert space (RKHS)) where function values can be expressed as a linear expansion of kernel functions. By using positive definite kernel functions, a convex loss function is guaranteed hence avoiding the problems with local minima [26]. The kernel least mean square (KLMS) algorithm [27] is a kernel-based online learning designed for online time series prediction via an adaptive learning process [28], [29] where the underlying pattern representations from time series data are extracted in a sequential manner.

Traditional mechanistic epidemiological models and the statistical and machine learning family of methods share a common feature; they all rely on a set of fixed pre-defined parameters and statistical assumptions. For example, the static reproduction number which is used in traditional mechanistic model has been singled out as the common cause of model failure in COVID-19 modeling [30]. A more dynamic and flexible approach is called for [3], [30] and with this expectation in mind, we are witnessing a new generation of epidemic models that combine mechanistic models with a dynamic measure of epidemic parameters that is driven by statistics and machine learning [31], [32].

In this paper, we propose a forecasting method for COVID19 that combines a data-driven dynamical system modelling with a dynamic nonlinear stochastic optimization strategy to adapt model parameters with data. The method is called the extrapolated kernel least mean square algorithm (extrap-KLMS) that uses memory-dependent gradient estimate to extrapolate future trends of epidemic state variables. Model training and verifications are done using COVID-19 epidemic data for Malaysia, Saudi Arabia and Italy in which we demonstrate the flexibility of forecasting future trends using a memory averaging window. A situational analysis of the pandemic in Malaysia is used as a case study to showcase the capabilities of extrap-KLMS. This paper is outlined as follows: the materials and methods is provided in Section II where we describe the underlying principles of data-driven modelling of dynamical system in the reproducing kernel Hilbert space (RKHS). In Section III, the training and validation process are described. Here we explain the role of each parameter in extrap-KLMS. In Section IV, situational analysis of the pandemic in Malaysia is given, with focus on the third wave of the pandemic.

\section{Materials And Methods}

Thorough discussions of the general principle of learning a dynamical system from sequence data can be found in [33] [36]. A continuously evolving dynamical system is commonly described as a system of differential equation of the form

$$
\frac{d \mathbf{x}}{d t}=\mathcal{F}(\mathbf{x}(t), \theta)
$$

where $\mathbf{x}(t) \in \mathbb{R}^{N}$ is the state of the system at time $t, \theta$ is a vector of parameters and the function $\mathcal{F}(\mathbf{x}(t), \theta)$ is a vector field that describes the dynamics of state evolution. The state is specified as a vector

$$
\mathbf{x}(t)=\left[x_{1}(t), x_{2}(t), \ldots, x_{N}(t)\right]^{T} \in V,
$$

where $V$ is an $\mathrm{N}$ dimensional manifold that is referred to as the state space and $x_{1}(t), x_{2}(t), \ldots, x_{N}(t)$ are scalar-valued time-dependent state variables. In the case of discrete-time, the state evolution is specified by discrete maps

$$
\mathbf{x}_{n+1}=\mathcal{F}\left(\mathbf{x}_{n}, \theta\right) \text {. }
$$

Here, $n$ denotes the discrete time step and the system is assumed to be sampled every $\Delta t$ in time such that the $n$th state vector $\mathbf{x}_{n}=\mathbf{x}\left(t_{0}+n \Delta t\right)$.

A general prediction model can be derived from (2) by building a parameterized nonlinear function $\mathcal{F}\left(\mathbf{x}_{n}, \theta\right)$ that takes $\mathbf{x}_{n}$ to $\mathbf{x}_{n+1}=\mathcal{F}\left(\mathbf{x}_{n}, \theta\right)$ and use suitable criterias to determine the parameters $\theta$. A common criteria is by the minimization of a loss function of the form

$$
L(\theta)=\left\|\mathbf{y}_{n}-\mathcal{F}\left(\mathbf{x}_{n}, \theta\right)\right\|^{2},
$$

where $\mathbf{y}_{n}$ is the concrete measured values of $\mathbf{x}_{n+1}$. Development of specific form of prediction model $\mathcal{F}\left(\mathbf{x}_{n}, \theta\right)$ can be based on several principles resulting in the family of BoxJenkins methods, artificial neural networks, fuzzy approaches, Bayesian and Support Vector Machine (SVM) techniques and many others.

\section{A. Prediction Model in the Reproducing Kernel Hilbert Space}

Suppose we have a set of scalar observations $s\left(t_{0}+n \Delta t\right)=$ $s_{n}$ of a discrete system which can be assumed as equally sampled data from one of the state variable of the system. Taken's delay embedding theorem [33] states that the geometric structure of the state space dynamics can be reconstructed from vectors of the form:

$$
\mathbf{y}_{n}=\left[s_{n}, s_{n-1}, \ldots, s_{n-M+1}\right]^{T} \in W,
$$

where the embedding dimension $M>2 N$. It is assumed that the Euclidean $M$-dimensional space $W$ is related to the original space of the state vector $\mathbf{x}_{n}$ by smooth, differentiable transformations such that in the neighbourhood of $\mathbf{y}_{n}, \mathbf{y}_{n+1}=\mathcal{F}_{W}\left(\mathbf{y}_{n}\right)$ where $\mathcal{F}_{W}\left(\mathbf{y}_{n}\right)$ is a local functional form for $\mathcal{F}\left(\mathbf{x}_{n}\right)$ reconstructed in the embedding space $W$. Suppose $\mathcal{F}_{W}\left(\mathbf{y}_{n}\right)=\left[f_{1}\left(\mathbf{y}_{n}\right), f_{2}\left(\mathbf{y}_{n}\right), \ldots, f_{M}\left(\mathbf{y}_{n}\right)\right]^{T}$ where each $f_{i}\left(\mathbf{y}_{n}\right), i=1,2, \ldots, M$ can be parameterized as follows:

$$
f_{i}\left(\mathbf{y}_{n}\right)=w_{i 1} \phi_{1}\left(\mathbf{y}_{n}\right)+w_{i 2} \phi_{2}\left(\mathbf{y}_{n}\right)+\ldots+w_{i M} \phi_{M}\left(\mathbf{y}_{n}\right) .
$$

As such, we write $\mathcal{F}_{W}\left(\mathbf{y}_{n}\right)=\mathbf{X} \Phi$ where

$$
\mathbf{X}=\left[\begin{array}{cccc}
w_{11} & w_{12} & \cdots & w_{1 M} \\
w_{21} & w_{22} & \cdots & w_{2 M} \\
\vdots & \vdots & \ddots & \vdots \\
w_{M 1} & w_{M 2} & \cdots & w_{M M}
\end{array}\right]
$$

Here we assume that

$$
\Phi\left(\mathbf{y}_{n}\right)=\left[\phi_{1}\left(\mathbf{y}_{n}\right), \phi_{2}\left(\mathbf{y}_{n}\right), \ldots, \phi_{M}\left(\mathbf{y}_{n}\right)\right]^{T},
$$


is a vector of known basis functions in $W$.

Consider the prediction of a future value of the time series, $s_{n+h}$ where $h=m \Delta t$ (for some integer $m$ ) is the prediction horizon and that the time series consists of equally sampled data from the $i$ th state variable $x_{i}(t)$. The embedding of $s_{n+h}$ on the coordinates associated with the local basis $\left\{\phi_{1}\left(\mathbf{y}_{n}\right), \phi_{2}\left(\mathbf{y}_{n}\right), \ldots, \phi_{M}\left(\mathbf{y}_{n}\right)\right\}$, takes the form $\Phi\left(\mathbf{y}_{n}\right)^{T} \mathbf{w}$ where the coefficient vector $\mathbf{w}=\left[w_{1}, w_{2}, \ldots, w_{M}\right]^{T}$ is determined by minimizing a loss function of the form

$$
L(\mathbf{w})=\min _{\mathbf{w} \in \mathbb{R}} \sum_{i=1}^{n}\left[s_{i+h}-\Phi\left(\mathbf{y}_{i}\right)^{T} \mathbf{w}\right]^{2} .
$$

Form the perspective of the state variable $x_{i}(t)=s(t)$ the reconstruction of $\mathcal{F}_{W}\left(\mathbf{y}_{n}\right)$ is also possible by interpreting the coordinates of $\mathbf{y}_{n}$ as successive temporal derivatives, i.e.,

$$
\mathbf{y}_{n}=\left[s(t), \frac{d s}{d t}, \frac{d^{2} s}{d t^{2}}, \frac{d^{3} s}{d t^{3}}, \ldots\right]^{T} .
$$

In the locality of $\mathbf{y}_{n}$, it is reasonable to approximate the first derivative as

$$
\frac{d s}{d t} \approx \frac{s(t+\Delta t)-s(t)}{\Delta t} .
$$

As such, the embedding of $s_{n+h}$ can also be interpreted as a forward euler step of the form

$$
s_{n+h} \approx s_{n}+\Delta t g_{n},
$$

where $g_{n}$ is a discrete approximation of the gradient of the state variable $s(t)$ at the $n$th time step. More generally, from the point of view of the state space,

$$
\mathbf{y}_{n+1}=\mathcal{F}_{W}\left(\mathbf{y}_{n}\right) \approx \mathbf{y}_{n+1}+\Delta t \mathbf{G}_{W}\left(\mathbf{y}_{n}\right)
$$

where $\mathbf{G}_{W}\left(\mathbf{y}_{n}\right)$ is the gradient field in the embedding space $W$.

The loss function in (3) requires explicit knowledge of the basis functions $\phi_{i}\left(\mathbf{y}_{n}\right), i=1,2, \ldots, M$. If such knowledge is not readily available, it is possible to define (3) based on only representations of $\phi_{i}\left(\mathbf{y}_{n}\right)$ in the form of kernels. In particular, we assume $\phi_{i}\left(\mathbf{y}_{n}\right), i=1,2, \ldots, M$ are nonlinear maps $\phi_{i}: \mathbb{R}^{M} \rightarrow W \subseteq \mathcal{H}$, where $\mathcal{H}$ is a RKHS. The similarity between the elements in $\mathcal{H}$ is measured using its associated inner product $(., .)_{\mathcal{H}}$ and it is computed via a kernel function $\kappa: \mathbb{R}^{M} \times \mathbb{R}^{M} \rightarrow \mathbb{R}$ such that $\left(\mathbf{y}_{k}, \mathbf{y}_{n}\right) \rightarrow \kappa\left(\mathbf{y}_{k}, \mathbf{y}_{n}\right)$. For positive definite kernel functions, we can ensure that [37] for all $\mathbf{y}_{k}, \mathbf{y}_{n} \in \mathbb{R}^{M}$,

$$
\left(\Phi\left(\mathbf{y}_{k}\right), \Phi\left(\mathbf{y}_{n}\right)\right)_{\mathcal{H}}=\kappa\left(\mathbf{y}_{k}, \mathbf{y}_{n}\right) .
$$

The property in (5) is called the 'kernel trick'. In RKHS, the loss function (3) can be written as

$$
L(\mathbf{w})=\sum_{i=1}^{n}\left[s_{i+h}-\left(\Phi\left(\mathbf{y}_{i}\right), \mathbf{w}\right)_{\mathcal{H}}\right]^{2} .
$$

It is also possible to write the loss function in (6) as

$$
L(\mathbf{w})=\|\mathbf{s}-\mathbf{K w}\|^{2}=\mathbf{s}^{T} \mathbf{s}-2 \mathbf{s}^{T} \mathbf{K} \mathbf{w}+\mathbf{w}^{T} \mathbf{K}^{T} \mathbf{K} \mathbf{w} .
$$

Here, $\mathbf{s}=\left[s_{1+h}, s_{2+h}, \ldots, s_{n+h}\right]^{T}$, is a vector of true observations and $\mathbf{K}=\left[\begin{array}{llll}\Phi\left(\mathbf{y}_{1}\right) & \Phi\left(\mathbf{y}_{2}\right) & \ldots & \Phi\left(\mathbf{y}_{n}\right)\end{array}\right]^{T}$. Matrix $\mathbf{G}=\mathbf{K}^{T} \mathbf{K}$ has entries $G_{i j}=\left(\Phi\left(\mathbf{y}_{i}\right), \Phi\left(\mathbf{y}_{j}\right)\right)_{\mathcal{H}}=\kappa\left(\mathbf{y}_{i}, \mathbf{y}_{i}\right)$ which is positive definite if the kernel function $\kappa(.,$.$) is$ positive definite. Therefore with a choice of a positive definite kernel function, the loss function in (7) is a convex function and the problem in (6) is a convex minimization. This is a clear advantage over other nonlinear machine learning technique for example neural network that are based on non-convex optimization and are very likely to have local minimas. Hence to guarantee a convex minimization problem, in the rest of the paper, we adopt the normalized Gaussian kernel, where

$$
\kappa(\mathbf{y}(i), \mathbf{y}(j))=e^{-\|\mathbf{y}(i)-\mathbf{y}(j)\|^{2} / 2 \sigma^{2}},
$$

where $\sigma>0$ is the kernel bandwidth.

\section{B. Kernel least mean square algorithm (KLMS)}

In dealing with various non-stationarities in the data, a dynamic regression approach can be employed where the loss function (7) is estimated using only the most recent data. A popular approach which has its roots in adaptive filtering [38], describes the current prediction of a time series at any particular time $n$ in the form $\left(\Phi\left(\mathbf{y}_{i}\right), \mathbf{w}\right)_{\mathcal{H}}$ and the estimated loss function is given by

$$
L_{\text {inst }}(\mathbf{w})=(e(n))^{2}=\left(s_{n+h}-\left(\Phi\left(\mathbf{y}_{n}\right), \mathbf{w}\right)_{\mathcal{H}}\right)^{2} .
$$

A stochastic gradient minimization of (9) searches for the optimum parameter vector $\mathbf{w}$ along the negative instantaneous gradient direction which is given by

$$
\nabla_{\mathbf{w}} L_{\text {inst }}(\mathbf{w})=2 e(n) \Phi\left(\mathbf{y}_{n}\right),
$$

where in (10), $e(n)=s_{n+h}-\left(\Phi\left(\mathbf{y}_{n}\right), \mathbf{w}(\mathbf{n}-\mathbf{1})_{\mathcal{H}}\right.$ is the apriori error.The update equation for $\mathbf{w}$ is then

$$
\mathbf{w}(n)=\mathbf{w}(n-1)+\mu e(n) \Phi\left(\mathbf{y}_{n}\right) .
$$

Assuming $\mathbf{w}(0)=\mathbf{0}$, it can be shown that [38]

$$
\mathbf{w}(n)=\mu \sum_{i=1}^{n} e(i) \Phi\left(\mathbf{y}_{i}\right) .
$$

Let the apriori prediction of $s_{n+h}$ be $\tilde{s}_{n+h}$ and it is given by:

$$
\begin{aligned}
\tilde{s}_{n+h} & =\left(\Phi\left(\mathbf{y}_{n}\right), \mathbf{w}(\mathbf{n}-\mathbf{1})_{\mathcal{H}}\right) \\
& =\mu \sum_{i=1}^{n-1} e(i)\left(\Phi\left(\mathbf{y}_{i}\right), \Phi\left(\mathbf{y}_{n}\right)\right)_{\mathcal{H}} \\
& =\mu \sum_{i=1}^{n-1} e(i) \kappa\left(\mathbf{y}_{i}, \mathbf{y}_{n}\right),
\end{aligned}
$$

where the last line in (13) is obtained by applying the kernel trick (5). It is clear from (13) that the apriori prediction can be computed using only knowledge of the kernel function. It follows that the aposteriori prediction of $s_{n+h}$ can be updated as follows:

$$
\hat{s}_{n+h}=\left(\Phi\left(\mathbf{y}_{n}\right), \mathbf{w}(\mathbf{n})_{\mathcal{H}}=\tilde{s}_{n+h}+\mu e(n) \kappa\left(\mathbf{y}_{n}, \mathbf{y}_{n}\right) .\right.
$$

The KLMS prediction model in (14) describes the h-steps ahead prediction of a time series as a linear expansion of an expanding set of kernel functions $\kappa\left(\mathbf{y}_{i}, \mathbf{y}_{n}\right), i=1,2, \ldots$, i.e., $\hat{s}_{n+h}=\sum_{i=1}^{n} \alpha_{i} \kappa\left(\mathbf{y}_{i}, \mathbf{y}_{n}\right)$ where each $\alpha_{i}=\mu e(i)$ is a 
model parameter that changes dynamical with each new information in the time series. A straightforward implementation of (9)-(14) leads to the Kernel Least Mean Square (KLMS) algorithm. The basic sequential rule for KLMS is given in Algorithm 1.

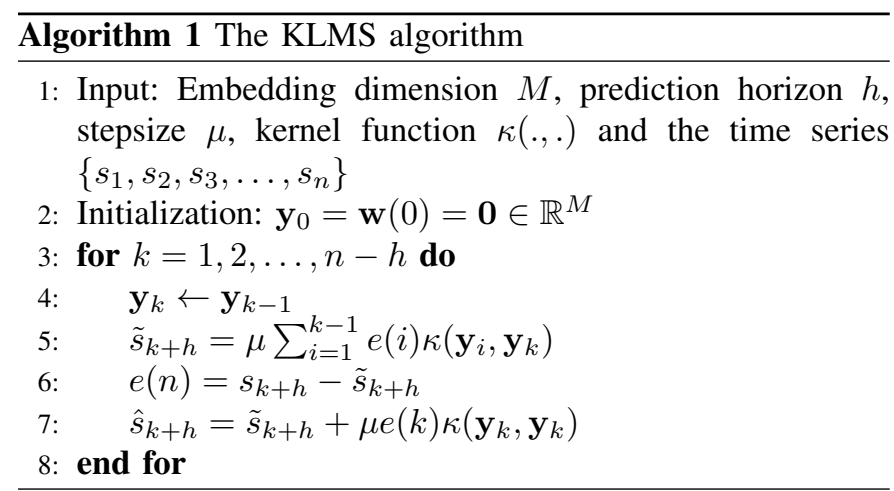

We refer readers to the convergence properties of KLMS as reported in [39], [40].

\section{Forecasting using KLMS: The extrapolated KLMS algo- rithm with memory}

The forecasting approach using KLMS is composed of two stages: i) the learning (training) stage, and ii) the forecasting stage. During the learning stage, KLMS learns a nonlinear prediction model $\hat{s}_{n}$ based on available training samples $\left\{\left(s_{1}, s_{1+h}\right),\left(s_{2}, s_{2+h}\right), \ldots,\left(s_{n-h}, s_{n}\right)\right\}$ and the computational procedures are as listed in Algorithm 1.In the forecasting stage, our objective is to estimate $\left\{s_{n+1}, s_{n+2}, \ldots, s_{n+h}\right\}$ while at the same time extending the nonlinear prediction model $\hat{s}_{n}$ over the prediction horizon $h$. The computation is based on the forward euler integration in (4), i.e.,

$$
\hat{s}_{n+i}=\hat{s}_{n+(i-1)}+\Delta t g_{i},
$$

where $\Delta t=1$ and the gradient of the state variable $g_{i}$ is estimated as the mean of the last $K$ instantaneous errors:

$$
g_{i}=\frac{1}{K} \sum_{l=1}^{K} e(n-l+i) \kappa\left(\mathbf{y}_{n-l-i}, \mathbf{y}_{n+l-i}\right)
$$

The parameter $K$ is the size of the averaging window used to estimate the current gradient $d s / d t$. The gradient estimate $g_{i}$ carries information on the last $K$ rates of change of the state variable at $K$ discrete times. In other words $g_{i}$ contains a memory of recent trends where $K$ determines the size of that memory. Therefore, the extrapolation step in (15) allows an extrapolation of the KLMS model by taking into account a memory of past trends. By varying $K$, we are able to extrapolate the model in a spectrum of different directions, guided by the memory-averaging window and the associated gradient estimate. The sequential rule for the so-called extrapolated KLMS (extrap-KLMS) algorithm with memory is listed in Algorithm 2.

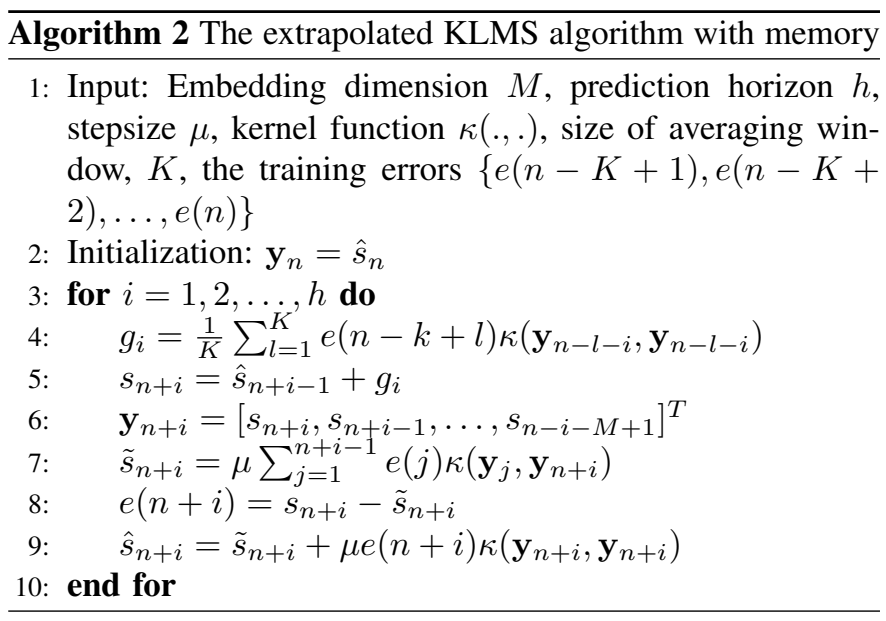

\section{TRAINING AND VALIDATION}

The effectiveness of KLMS in capturing essential features of the COVID-19 pandemics and in forecasting future trends are validated using a series of experiments, details of which are given in this section. The experiments are divided into two stages; the training stage and the testing (forecasting) stage. Predictive performance of KLMS algorithm is mostly determined by the choice of two parameters: the kernel bandwidth, $\sigma$ and the step-size $\mu$. In the training stage, optimal choices of these parameters for a given dataset are determined. The mean square error (MSE) is used to assess optimality in both training and testing stage. Training MSE is defined by

$$
E_{\text {train }}=\frac{1}{n} \sum_{i=1}^{n} e(i)^{2},
$$

( $n$ is the number of training samples) and test MSE is given by

$$
E_{\text {test }}=\frac{1}{h} \sum_{i=1}^{h} e(n+i)^{2},
$$

( $h$ is the prediction horizon which is also the number of test samples).

\section{A. Datasets}

Data used in the training and validation process are taken from https://www.worldometers.info/coronavirus/ and we have used the time series data of three different countries in this study: Malaysia, Saudi Arabia and Italy. Experiments are performed based on the following time series:

1) Number of active cases;

2) Number of removed case (total recoveries + total deaths).

The number of active (infected) and removed cases are the state variables in the standard S-I-R model, thus it makes sense to tune the model parameters to these data sets. Data sets are taken for the dates February 15, 2020 to January 28, 2020. Each data set is split into training and testing (see Section III-C) and scaled into the range of $[0,1]$. The errors $e($.$) which$ are used in the computation of training and test MSE are based on the scaled data. 


\section{B. Choice of parameters}

There are two types of parameters that require tuning and these are i) the kernel bandwidth, $\sigma$; ii) the KLMS step-size, $\mu$; In this section we will describe the role of each parameter in the KLMS prediction model and how to determine the optimal parameter values empirically.

1) Effects of kernel bandwidth $\sigma$ : With the employment of the Gaussian kernel, there is one parameter that needs to be tuned and that is the kernel bandwidth. The kernel bandwidth, $\sigma$ helps define the inner product $(., .)_{\mathcal{H}}$ which is the metric of similarity in RKHS. If the kernel bandwidth is too large, then all the data would look similar in the RKHS (with inner products all close to 1), and the system reduces to linear regression. If the kernel bandwidth is too small, then all the data would look distinct (with inner products all close to 0 ) and the system cannot do inference on unseen samples that fall between the training points. Choosing an appropriate kernel bandwidth for each dataset is important to ensure KLMS gives an optimal performance.

In Figure 1 (left), several example of trained models for the number of active cases for Malaysia, Saudi Arabia and Italy respectively are presented to show the effect of different values of $\sigma$ on the quality of the model. For all the time series for the three countries, with a small value of $\sigma=0.01$, only a limited feature of the time series is captured. As $\sigma$ is increased, the prediction model increases in quality until $\sigma$ reaches an optimum value. Increasing $\sigma$ beyond the optimum value does not result in any improvement in the quality of the prediction model. In Figure 1 (right), the trained models for $\sigma=10$ are shown. For this value of $\sigma$, a very good fit to the data is obtained for Malaysia, Saudi Arabia and Italy where all the distinct features of the time series have been well captured.

Training MSE for a range of values for $\sigma$ are produced in Table I. We can see from Table I that the MSE values achieve a minimum when $\sigma=10$ and remain around the same value as $\sigma$ is increased. Results in Figure 1 and Table I are produced using $\mu=1$ and $h=14$ where $h$ is the prediction horizon. A thorough investigation has revealed that these results are independent of the choice of $\mu$ and $h$.

2) Effects of step-size $\mu$ : Another important parameter to tune is the step-size used in the KLMS algorithm for both training and testing. The form of the KLMS update equation (update) suggests that KLMS updates the state variable from one state to the other via a forward Euler step where $\mu$ is the time-step in the numerical integrator. It can be seen that the step-size $\mu$ plays the role of a time-step. Hence it provides a trade-off between stability and accuracy.

In Figure 2 (left), several example of trained models for the number of active cases for Malaysia, Saudi Arabia and Italy respectively are presented to show the effect of different values of $\mu$ on the prediction model. For all the time series for the three countries, with a small value of $\mu=0.001$, the captured profile is rather flat that suggests the inability of KLMS prediction model to capture significant dynamics of the time series. Significant improvement in the quality of the prediction model is observed as $\mu$ is increased to 0.1 and greater. However as $\mu$ reaches the value of 2 , signs of instability begin to appear. In Figure 2 (right), the trained models for the value $\mu=1$ is included which shows a very good agreement with the actual data for Malaysia, Saudi Arabia and Italy.

\section{Forecasting using extrap-KLMS with Memory}

It is evident from Figure 1 and Figure 2 that a single value of $\sigma$ (and $\mu$ ) can be applied to the entire training samples to produce an optimum model. Henceforth, we will be using the values $\sigma=10$ and $\mu=1$ to train the KLMS model. Once a trained model is obtained, the performance of extrapKLMS can be tuned further using the parameter $K$ which is the size of the averaging window that is used to compute the gradient estimate for extrapolation. The choice of $K$ depends very much on how many past events that need to be included to produce the gradient estimate. For the forecasting of COVID19 , understanding the timeline of the pandemic is important to decide the value to use for $K$ and to interpret the ensuing predicted future trends. As a general rule, a small $K$ is used if only the most recent events are expected to influence future trends. Whereas, if there are past events that are associated with clusters of infection that are still active, or a lot of undetected cases is expected, choosing a larger $K$ value may be more appropriate.

Experiments in this section are conducted using data for the number of active cases for Malaysia, Saudi Arabia and Italy. Each experiments is performed using a segment of a time series of length $n+h$ where $n$ refer to the number of samples used for training and $h$ refer to the number of samples for testing the accuracy of forecasts. Here $n$ denotes the number of days since February 15, 2020 and $h$ represents the forecasting horizon, i.e., the forecasts are computed for days $n+1, n+2, \ldots, n+h$. In all the experiments in this section, $h$ is set to 14 . The period of 14 days corresponds with the incubation period (time from exposure to the development of symptoms) of COVID-19 which is estimated to be 14 days [41], [42]. Figures 3-5 show examples of 14-days ahead forecasting of the number of active cases in Malaysia, Saudi Arabia and Italy. For each country, two different experiments are performed involving two different values of $n$.

If we observe the profile of the actual data for Malaysia in Figure 3, from $n=50$ to $n=140$, the number of active cases is showing an overall decreasing trend apart from two instances where localized clusters appear. The first localized clusters see the number of active cases plateauing from $n=73$ to $n=80$, however this trend does not persist. The second localized cluster see a slight increase in the number of cases from $n=100$ to $n=115$. Similar to the first localized cluster, the growth pattern of the second cluster also does not persist. We performed two experiments to demonstrate how to differentiate non-persistent trends from persistent trends. In Figure 3(a) a spectrum of 14-days ahead forecast is produced at $n=80$ to analyse the short-term future trends captured by extrap-KLMS in the locality of the first localized cluster. The spectrum consists of forecasts computed using $K=7,14,21,28$. The maximum averaging window with $K=28$ captures all the events from $n=51$ to $n=80$. With $K=7$, the 14-days ahead forecast produced 


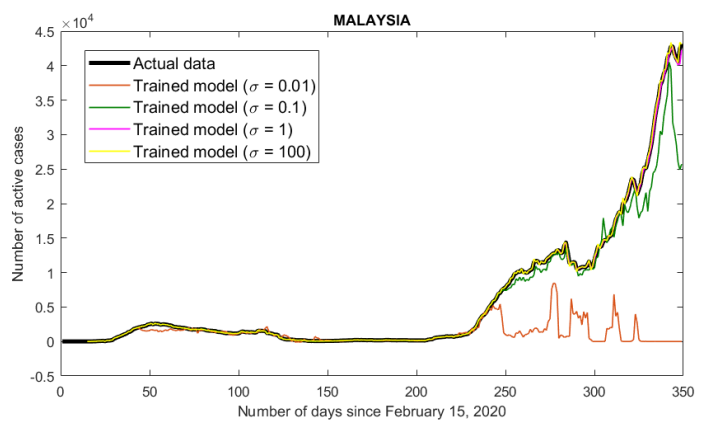

(a)

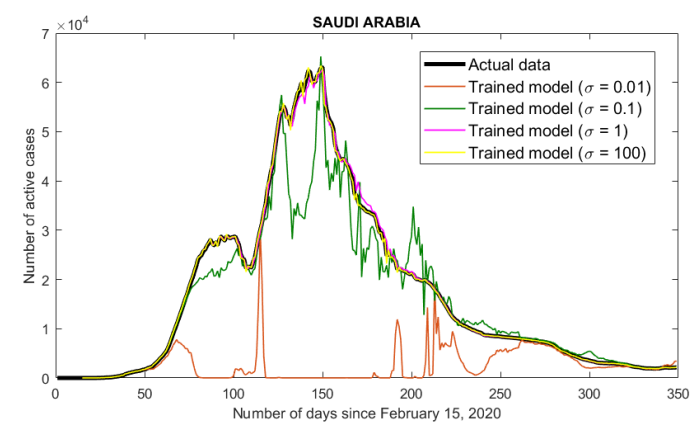

(c)

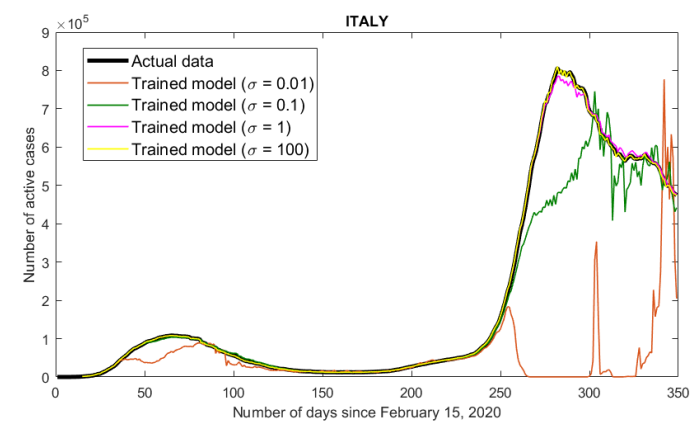

(e)

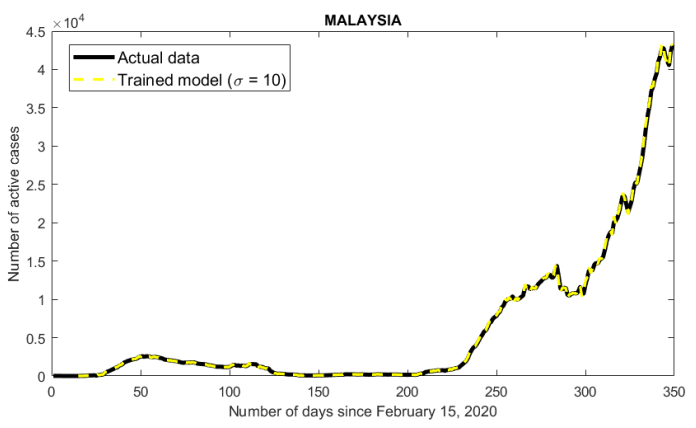

(b)

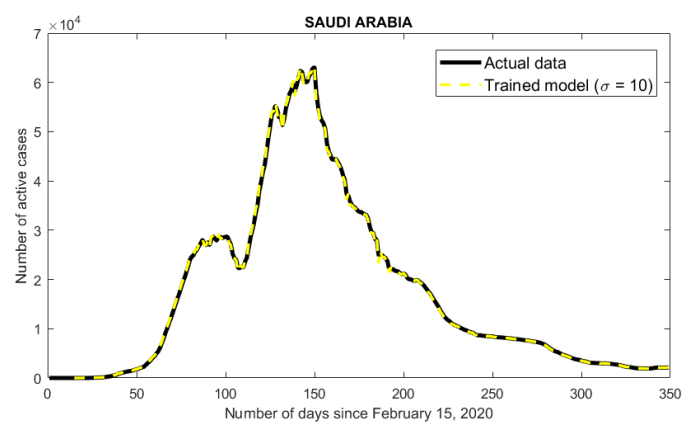

(d)

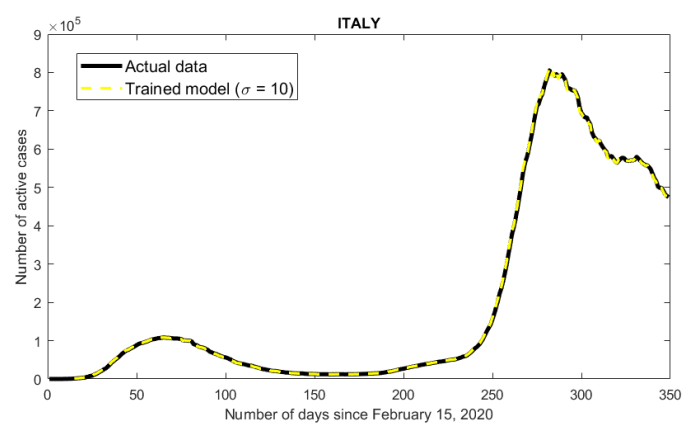

(f)

Fig. 1: Effects of different kernel bandwidth on the quality of prediction model.

TABLE I: Training MSE for different values of kernel bandwidth, $\sigma$

\begin{tabular}{|c|c|c|c|c|c|c|}
\hline$\sigma$ & Malaysia (Active) & Malaysia (Removed) & S.Arabia (Active) & S.Arabia (Removed) & Italy (Active) & Italy (Removed) \\
\hline 0.01 & $6.80 \times 10^{-2}$ & $6.69 \times 10^{-1}$ & $1.40 \times 10^{-1}$ & $7.86 \times 10^{-2}$ & $1.35 \times 10^{-1}$ & $9.19 \times 10^{-2}$ \\
\hline 0.1 & $8.90 \times 10^{-3}$ & $4.70 \times 10^{-3}$ & $1.21 \times 10^{-2}$ & $2.10 \times 10^{-3}$ & $1.02 \times 10^{-2}$ & $3.90 \times 10^{-3}$ \\
\hline 0.5 & $6.34 \times 10^{-4}$ & $3.24 \times 10^{-4}$ & $1.00 \times 10^{-3}$ & $1.33 \times 10^{-4}$ & $8.10 \times 10^{-4}$ & $2.50 \times 10^{-4}$ \\
\hline 1 & $2.49 \times 10^{-4}$ & $1.19 \times 10^{-4}$ & $4.41 \times 10^{-4}$ & $5.18 \times 10^{-5}$ & $3.05 \times 10^{-4}$ & $9.14 \times 10^{-5}$ \\
\hline 5 & $1.03 \times 10^{-4}$ & $3.61 \times 10^{-5}$ & $1.77 \times 10^{-4}$ & $2.10 \times 10^{-5}$ & $9.70 \times 10^{-5}$ & $2.95 \times 10^{-5}$ \\
\hline 10 & $9.86 \times 10^{-5}$ & $3.34 \times 10^{-5}$ & $1.69 \times 10^{-4}$ & $2.00 \times 10^{-5}$ & $9.06 \times 10^{-5}$ & $2.76 \times 10^{-5}$ \\
\hline 15 & $9.79 \times 10^{-5}$ & $3.29 \times 10^{-5}$ & $1.68 \times 10^{-4}$ & $1.98 \times 10^{-5}$ & $8.94 \times 10^{-5}$ & $2.72 \times 10^{-5}$ \\
\hline 20 & $9.76 \times 10^{-5}$ & $3.27 \times 10^{-5}$ & $1.67 \times 10^{-4}$ & $1.98 \times 10^{-5}$ & $8.90 \times 10^{-5}$ & $2.71 \times 10^{-5}$ \\
\hline 25 & $9.75 \times 10^{-5}$ & $3.27 \times 10^{-5}$ & $1.67 \times 10^{-4}$ & $1.98 \times 10^{-5}$ & $8.89 \times 10^{-5}$ & $2.70 \times 10^{-5}$ \\
\hline 30 & $9.74 \times 10^{-5}$ & $3.26 \times 10^{-5}$ & $1.67 \times 10^{-4}$ & $1.98 \times 10^{-5}$ & $8.87 \times 10^{-5}$ & $2.70 \times 10^{-5}$ \\
\hline
\end{tabular}

TABLE II: Training MSE for different values of step-size, $\mu$

\begin{tabular}{|c|c|c|c|c|c|c|}
\hline$\mu$ & Malaysia (Active) & Malaysia (Removed) & S.Arabia (Active) & S.Arabia (Removed) & Italy (Active) & Italy (Removed) \\
\hline 0.001 & $6.75 \times 10^{-2}$ & $7.08 \times 10^{-2}$ & $1.36 \times 10^{-1}$ & $3.83 \times 10^{-1}$ & $1.51 \times 10^{-1}$ & $9.83 \times 10^{-2}$ \\
\hline 0.01 & $3.57 \times 10^{-2}$ & $3.43 \times 10^{-2}$ & $7.29 \times 10^{-2}$ & $8.70 \times 10^{-2}$ & $6.99 \times 10^{-2}$ & $4.32 \times 10^{-2}$ \\
\hline 0.1 & $3.38 \times 10^{-3}$ & $2.12 \times 10^{-3}$ & $6.32 \times 10^{-3}$ & $1.79 \times 10^{-3}$ & $5.88 \times 10^{-3}$ & $2.33 \times 10^{-3}$ \\
\hline 0.5 & $2.66 \times 10^{-4}$ & $1.25 \times 10^{-4}$ & $4.92 \times 10^{-4}$ & $7.78 \times 10^{-5}$ & $3.29 \times 10^{-4}$ & $1.08 \times 10^{-4}$ \\
\hline 1.0 & $1.02 \times 10^{-4}$ & $3.44 \times 10^{-5}$ & $1.75 \times 10^{-4}$ & $2.09 \times 10^{-5}$ & $9.28 \times 10^{-5}$ & $2.86 \times 10^{-5}$ \\
\hline 1.5 & $7.23 \times 10^{-5}$ & $1.70 \times 10^{-5}$ & $1.28 \times 10^{-4}$ & $1.08 \times 10^{-5}$ & $5.19 \times 10^{-5}$ & $1.42 \times 10^{-5}$ \\
\hline 2.0 & $7.56 \times 10^{-4}$ & $4.51 \times 10^{-5}$ & $5.50 \times 10^{-3}$ & $7.87 \times 10^{-5}$ & $1.06 \times 10^{-4}$ & $3.04 \times 10^{-5}$ \\
\hline 2.5 & $1.22 \times 10^{107}$ & $4.02 \times 10^{107}$ & $3.44 \times 10^{104}$ & $3.06 \times 10^{102}$ & $1.66 \times 10^{109}$ & $4.67 \times 10^{106}$ \\
\hline
\end{tabular}




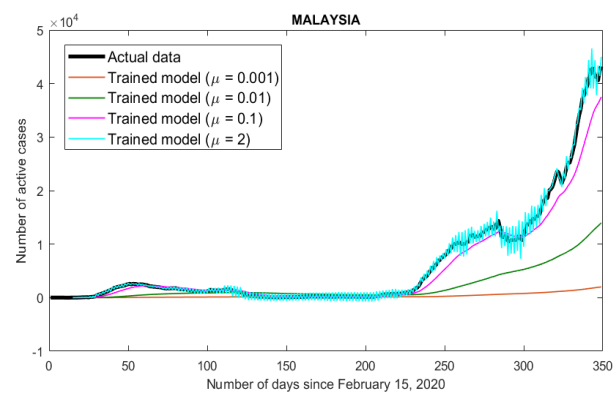

(a)

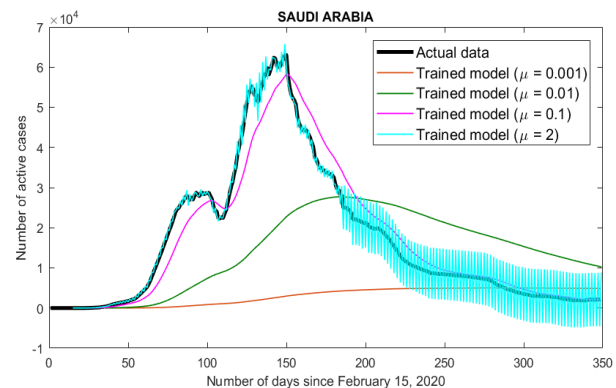

(c)

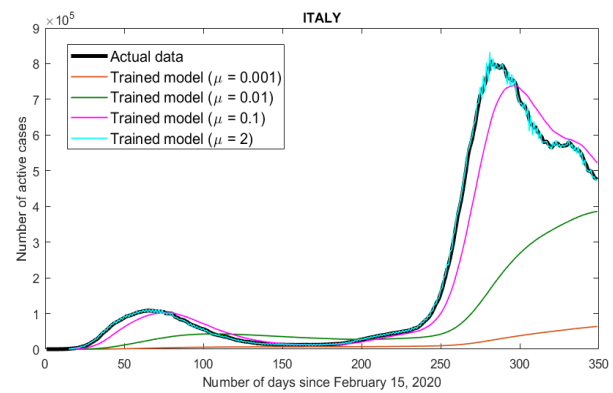

(e)

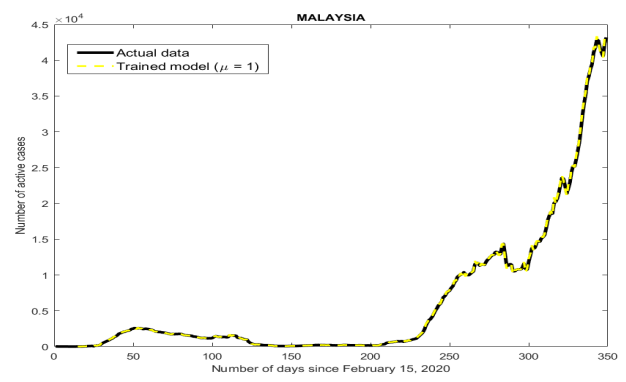

(b)

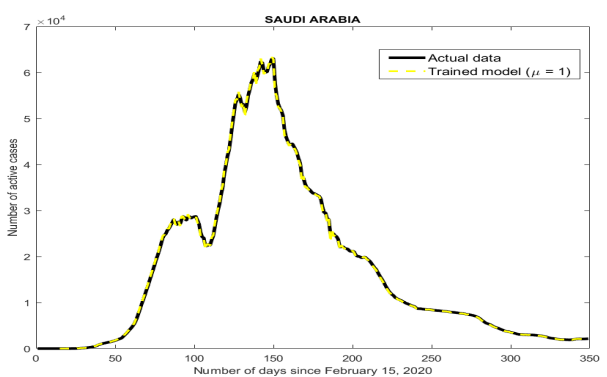

(d)

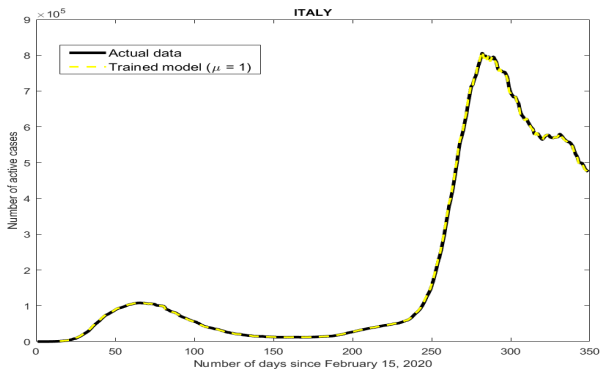

(f)

Fig. 2: Effects of different step-size on the quality of prediction model.

follow the non-persistent plateauing trend of the time series. As we increase the value of $K$, the 14-days ahead forecast start to approach a more persistent trend which is consistent with the long-term trend of the time series. In Figure 3(b) a spectrum of 14-days ahead forecast is produced at $n=112$ to analyse the short-term future trends in the locality of the second localized cluster. The spectrum consists of forecasts computed using $K=7,14,21,28,42,56,63$. With $K=7$, the 14-days ahead forecast depicts an increasing trend that follows the most recent trend of the time series. As $K$ is increased the trend of the forecast changes from increasing to decreasing and finally settle on a persistent trend that follows the overall long-term trend of the time series. Both experiments above demonstrate that a non-persistent pattern in the time series will lead to a non-persistent trend in the forecast as $K$ is increased. A particular trend is persistent when the size of the memory averaging window is large enough to capture all the independent events in the past.

The first experiment conducted on the Saudi Arabia data involves data from February 15, 2020 to May 14, 2020 ( $n=$ 90). In Figure 4(a), it is shown that this part of the time series correspond to the early outbreak of the pandemic in Saudi
Arabia which sees an increase in the number of active cases. The rate of increase reduces when $n=80$ and the curve flattens from $n=88$ to $n=100$. We produce a spectrum of 14-days ahead forecast for $K=7,14,21,28,35$ with a maximum averaging window of $K=35$. It is observed that the 14-days ahead forecast for $K=7$ provide better prediction of short-term future values of the time series compared to the forecasts for the other values of $K$ (it also gives the smallest test MSE). As we increase $K$, the trends show a steeper rate of increase and then they remain relatively unchanged when $K=$ $21,28,35$. The changing trends of the forecasts give indication that the trend captured for $K=7,14$ are non-persistent and may be due to various nonstationarities in the observed data. The trend for the larger $K$ values are more consistent with the long-term trend of the time series. This goes to show that, a good short-term forecast may not necessarily entail a good generalization to the long-term future trend. In this experiment we highlight the flexibility of extrap-KLMS, whereby the value of $K$ can be used to predict different scenarios of future trend depending on the number of past events that we choose to incorporate in the gradient estimate. The ability to observe future trends from several different scenarios will allow better 


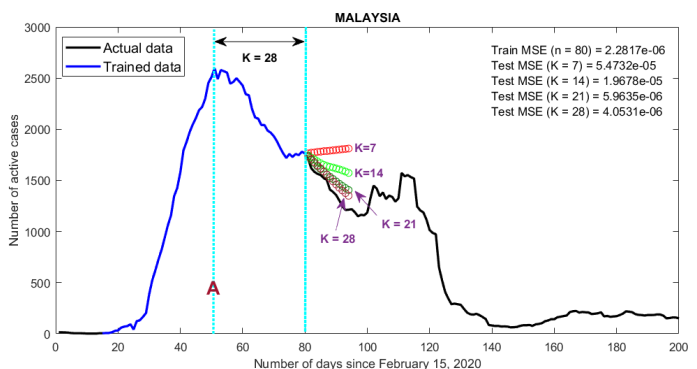

(a)

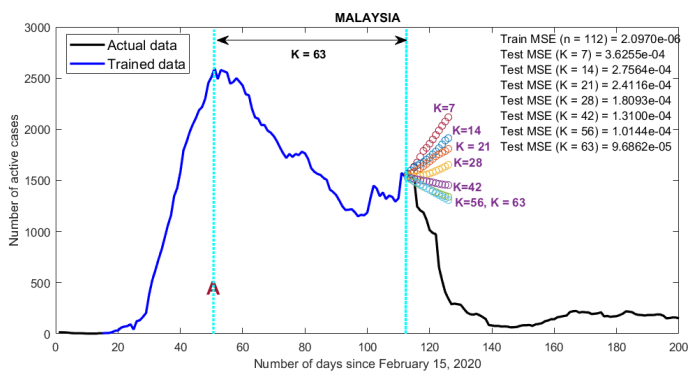

(b)

Fig. 3: Examples of short-term forecasting of Malaysian data using extrap-KLMS.

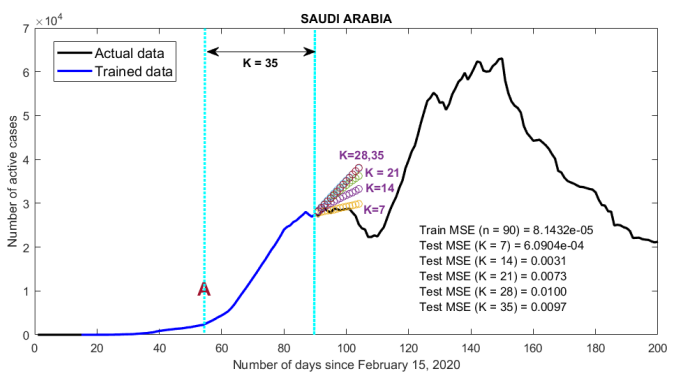

(a)

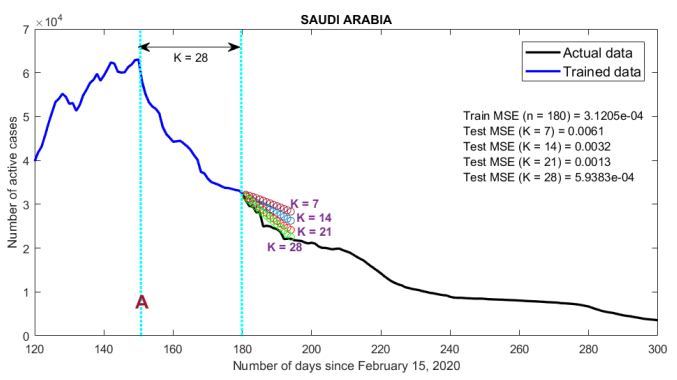

(b)

Fig. 4: Examples of short-term forecasting of Saudi Arabian data using extrap-KLMS.

generalization of the the longer term behaviour of the time series.

Figure 4(b) presents the result of the second experiment on the Saudi Arabia data. In this case, we focus on the portion of the time series from $n=152$ and $n=180$. The number of active cases is clearly decreasing and the trend is fairly stationary. We produce a spectrum of 14-days ahead forecast for $K=7,14,21,28$ and for all values of $K$ a persistent pattern is observed in the forecasts. As $K$ is increased the trend of the forecasts do not vary a lot. This experiment shows us that, when the current trend of the time series is stationary, changing the value of $K$ will not result in a vastly different trends in the forecasts.

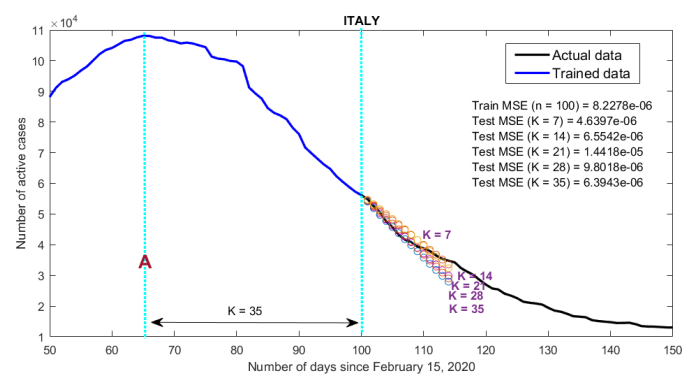

(a)

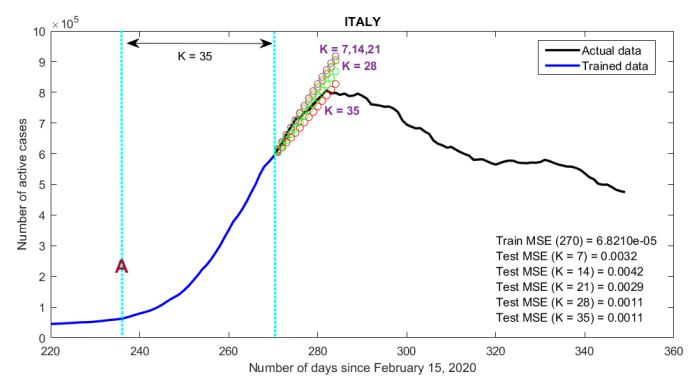

(b)

Fig. 5: Examples of short-term forecasting of Italian data using extrap-KLMS.

The experiments performed on Italy data are shown in Figure 5(a) (with $n=100$ ) and Figure 5(b) (with $n=270$ ). In both cases the 14-days ahead forecast with all the chosen values of $K$ show almost exactly the same short-term future trend. This suggests that the predicted trends are persistent. We observe that the averaging windows for all $K$ values cover events which are mostly stationary, thus resulting in a short-term predictions that fit well with data The predictions in Figure 5(a) are also consistent with the long-term trend of the time series. However the predictions in Figure 5(b), are unable to predict the changing trend occurring around $n=280$. This changing trend coincide with the decision by the Italian authorities to tighten up measures from November 05, 2020 onwards [43]. In this particular scenario, future events introduce new trends and features that are not yet learned by extrap-KLMS, thus its performance is only as good as can be expected from any other machine learning technique.

\section{Situational Analysis: Malaysia}

In this section, the extrap-KLMS is used to produce a detailed situational analysis for the COVID-19 pandemic in Malaysia. The objective is to investigate the capabilities of extrap-KLMS to explain certain responses of the COVID-19 pandemic with respect to historical events and various nonpharmaceutical interventions (NPI) taken by the authorities. The data set used in the experiments in this section is the number of daily new cases from February 15, 2020 to February 
TABLE III: Timeline of significant events in Malaysia from September 2020 to January 2021

\begin{tabular}{|l|l|}
\hline Dates & Events \\
\hline $\begin{array}{l}\text { September 2020 } \\
\text { September 26, 2020 } \\
\text { October 01, 2020 }\end{array}$ & $\begin{array}{l}\text { Spikes in COVID-19 cases in Sabah } \\
\text { The Sabah state election } \\
\text { Tembok cluster and politicians coming back from } \\
\text { Sabah }\end{array}$ \\
October 12, 2020 & CMCO in Klang Valley \\
November 07, 2020 & $\begin{array}{l}\text { CMCO in most part of Peninsular Malaysia (no } \\
\text { inter-state travel) } \\
\text { Outbreak at Top Glove factories (Teratai Cluster) } \\
\text { November 16, 2020 } \\
\begin{array}{l}\text { Inter-state travel was allowed nationwide } \\
\text { January 13, 2021 }\end{array}\end{array}$ \\
\hline
\end{tabular}

11, 2021 however the situational analysis is restricted to the events from September 15, 2020 to February 11, 2021 which corresponds to the third wave of COVID-19 pandemic in Malaysia. All experiments are performed using $\sigma=10$ and $\mu=1$.

\section{A. Timeline of significant events}

There are several important events that shape the severity of the third wave of COVID-19 in Malaysia. The first being the Sabah state election which saw a large number of politicians from Peninsular Malaysia travelled to Sabah and back [44]. At the time of the election there was already a surge in the number of cases believed to be caused by illegal immigrants entering Sabah [46]. Massive movements during election campaign and low adherence to standard operating procedures (SOPs) aggravated the transmissibility of COVID19 in Sabah [44], [45]. The second important event was the outbreak among construction and factory workers in Selangor [47]-[49]. Another significant event is when the restriction of inter-state travel was lifted on December 07, 2020 [50], after it was initially disallowed during the Conditionally Movement Control Order (CMCO) that was imposed on November 07, 2020. A full Movement Control Order (MCO2) was reinstated on January 13, 2020 when the number of daily cases started to reach more than 3000 cases. A timeline of significant events that are believed to have contributed to the third wave of COVID-19 in Malaysia is provided in Table III.

\section{B. Situational analysis before $\mathrm{MCO} 2$}

Figure 6 analyses the impact of the Sabah state election on COVID-19 pandemic in Malaysia from four perspectives:

1) the situation immediately after the election: We use $n=$ 230 with training samples from February 15, 2020 to October 01, 2020 to produce 90-days ahead forecast. The expected future trend is computed using averaging window of $K=30$ to capture persistent trends from events in the month of September, 2020.

2) the situation immediately after the imposition of $\mathrm{CMCO}$ in Klang valley: We use $n=244$ with training samples from February 15, 2020 to October 14, 2020 to produce 90-days ahead forecast. The expected future trend is computed using averaging window of $K=44$ to capture persistent trends from events in the month of September, 2020 and the events after the election.

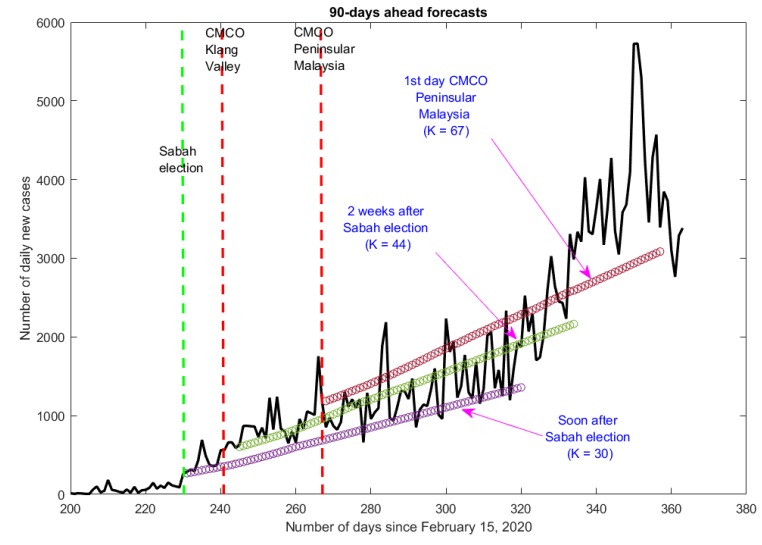

Fig. 6: Forecast after Sabah election.

3) the situation on the 1st day of the imposition of CMCO in Peninsular Malaysia: We use $n=267$ with training samples from February 15, 2020 to November 07, 2020 to produce 90-days ahead forecast. The expected future trend is computed using averaging window of $K=67$ to capture persistent trends from events in the month of September, 2020, the events after the election, the effects of CMCO in Klang valley and the events leading to $\mathrm{CMCO}$ in Peninsular Malaysia.

The 90-days ahead forecast for $n=230$ shows an increasing trend but the persistent feature of the third wave is not yet captured at this stage. The first major persistent pattern is captured in the 90-days ahead forecast for $n=244$, most likely due to massive movements during election campaign, followed by the return of politicians to Peninsular Malaysia and initiated new clusters of infections in various parts of Peninsular Malaysia. The CMCO in Peninsular Malaysia was imposed at a timely manner because the 90-days ahead forecast for $n=267$ depicts that a much faster growth in the number of daily cases is expected and the predicted trend is also consistent with the long-term outlook of the pandemic. The persistent increasing trends starting from October 01, 2020 provide a strong indicator that the third wave of the pandemic was mostly contributed by the events surrounding the Sabah election [44], [45].

Figure 7 analyses the impact of $\mathrm{CMCO}$ which was reinstated in most part of Peninsular Malaysia beginning November 07, 2020. Four sets of forecast are produced;

1) two weeks after $\mathrm{CMCO}(n=280)$ : Involves training samples from February 15, 2020 to November 20, 2020. The size of averaging window is $K=50$ and this covers events from October 01, 2020 to November 20, 2020. During this time, $\mathrm{CMCO}$ was in full swing and interstate travel was very restrictive.

2) three weeks after CMCO $(n=287)$ : Involves training samples from February 15, 2020 to November 27, 2020. The size of averaging window is $K=57$ and this covers events from October 01, 2020 to November 27, 2020. This period witness the lifting of $\mathrm{CMCO}$ restrictions in several states. A surge of the number of cases was 


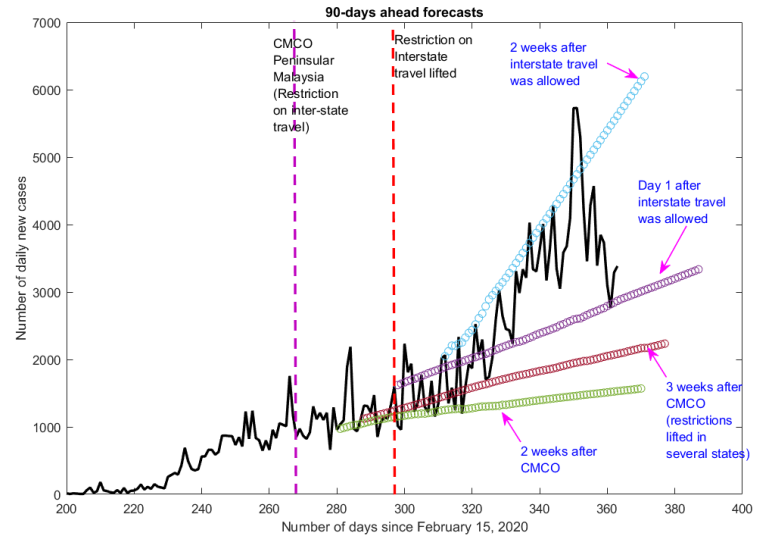

Fig. 7: Forecast leading to $\mathrm{MCO} 2$.

also reported during this time involving the Top Glove dormitories (or Teratai) cluster.

3) December 07, 2020 ( $n=297)$ : Involves training samples from February 15, 2020 to December 07, 2020. The size of averaging window is $K=67$ and this covers events from October 01, 2020 to December 07, 2020. During this period, a number of work-related clusters began to emerge and December 07, 2020 marked the first day of the lifting of inter-state travel restriction.

4) December 20, $2020(n=310)$ : Involves training samples from February 15, 2020 to December 20, 2020. The size of averaging window is $K=80$ and this covers events from October 01, 2020 to December 20, 2020.The timing of the forecast coincides with two weeks after the restrictions on inter-state travel was lifted.

From Figure 7, it is observed that the first 90-days ahead forecast clearly exhibits a lowering rate of increase in the number of cases. This suggests that the $\mathrm{CMCO}$ restrictions were successful in taming the pandemic at this stage. However, the second, third and fourth 90-days ahead forecast start to paint a totally different picture. A gradual hike in the rate of increase of daily cases is observed and the increasing rate is mostly accentuated by the ease of CMCO restrictions. In summary, from the results in Figure 7 , we can conclude that measures taken during CMCO in Peninsular Malaysia did not result in a persistent trend that can change the course of the pandemic.

\section{Situational analysis during $\mathrm{MCO} 2$}

The second Movement Control Order (MCO2) was imposed on January 13, 2021 which saw tougher restrictions compared to the $\mathrm{CMCO}$. The impact of $\mathrm{MCO} 2$ is analysed by producing 60 -days ahead forecast on Day $1(n=334)$, Day $8(n=341)$, Day $15(n=348)$, Day $22(n=355)$ and Day $29(n=362)$ of MCO2. These forecast will allow us to see how the trends develop on a weekly basis. We present the different perspectives of the analysis and these perspectives depend on the size of the averaging window $K$. In Figure 8, the forecasts are computed using averaging windows that cover events up to 15 days before the imposition of $\mathrm{MCO} 2$ whereas in Figure

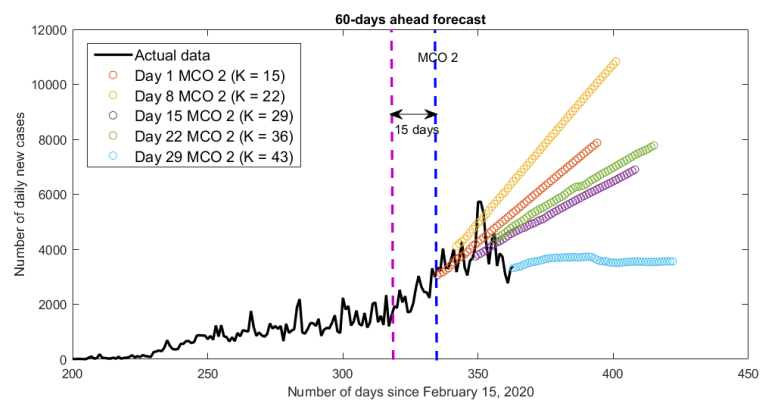

Fig. 8: Averaging window covers 15 days before MCO 2.

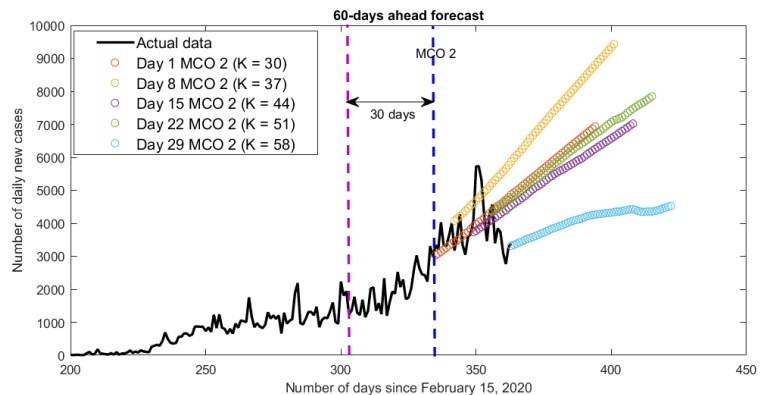

Fig. 9: Averaging window covers 30 days before MCO 2.

9, the forecasts are computed using averaging windows that cover events up to 30 days before the imposition of MCO2. In both cases, it can be seen that rate of increase of daily cases increases for the first week and only starts to decrease in the second week of MCO2. Plateauing of the number of daily cases is predicted to occur in week 4. Careful observation of the forecasts in week 4 reveals that, the shorter averaging window produces predicted number of daily cases plateauing around 3000 cases per day. On the other hand, with the longer averaging window, the predicted number of cases plateau around 4000 cases per day. This plateauing phenomenon is consistent with the results in [51] where COVID-19 plateau is observed when adaptive prevention strategies (prevention strategies with varying intensities) are used. The theoretical results in [51] are corroborated with real-world data from countries such as Thailand, South Korea, Tajikistan and Qatar.

In order to see more clearly the effect of the size of the averaging window on the trends after week 4 of $\mathrm{MCO} 2$, we produce 60-days ahead forecasts on Day 29 with different values of $K$ with the following details:

- $K=28$ covering events from January 13, 2020 to February 10,2021

- $K=43$ covering events from December 30, 2020 to February 10,2021

- $K=58$ covering events from December 16, 2020 to February 10,2021

- $K=73$ covering events from December 02, 2020 to February 10,2021

- $K=88$ covering events from November 18, 2020 to February 10,2021

It can be observed from Figure 10 that for $K=28$ (i.e. if only events during $\mathrm{MCO} 2$ are considered), a plateauing of 


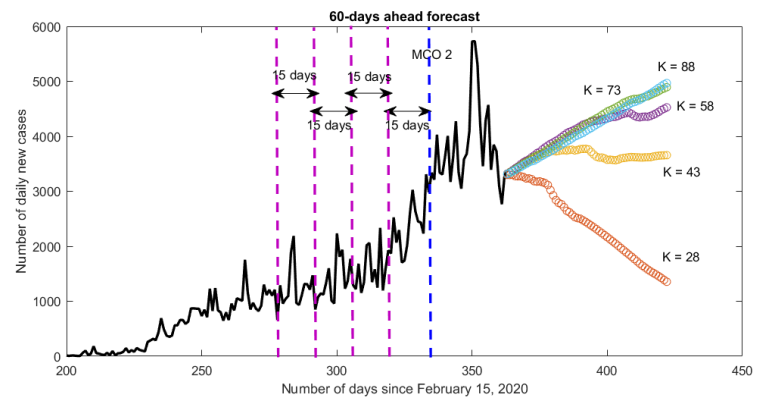

Fig. 10: Forecasts after week 4 of MCO 2 for different values of $K$.

the number of cases is immediately seen after week 4 of $\mathrm{MCO} 2$ followed by a decrease in the number of cases. For $K=43$ (i.e. include events during $\mathrm{MCO} 2$ and 15 days before that), the number of daily cases plateau between 3000 to 4000 cases daily. For $K=58$ an increasing trend is observed and this trend persists for $K=73$ and $K=88$. From these observation, we can deduce that the trends depicted for $K=28$ and $K=43$ are short-term trends which may not persist in the long run. This is because there exists earlier events included in $K=58,73,88$ that are still active and providing energy to the pandemic. A plateauing of the number of daily cases is still possible but this will occur beyond the 60 days where the number of daily cases will reach just above 5000 cases daily.

\section{CONCLUSION}

The extrap-KLMS algorithm with memory is a method that combines data-driven dynamical system modelling with a dynamic nonlinear stochastic optimization strategy. The learning process involves reconstruction of the phase space of the dynamical system in a reproducing kernel Hilbert space where defining features of the dynamical system are learned and expressed in the form of kernel functions. The prediction model of extrap-KLMS is a linear expansion of an expanding set of kernel functions and the model parameters change dynamically with each new data in the time series of the state variable. Future trends are computed by extrapolating the prediction model using a forward euler step along the direction of a memory-dependent estimate of the gradient of the state variable in question. The so-called 'memory' refers to the averaging window used for the gradient estimate. The size of the averaging window determines how many past events are included in the gradient estimation. In Section III-C and Section IV, the role of the size of averaging window, $K$, is demonstrated. We summarize the key points as follows:

1) When analysing a new trend in the time series (see Figure 3, $K$ can be used to investigate whether or not the trend will persist. A persistent trend usually does not vary too much with $K$;

2) In a nonstationary situation where there is constantly changing trends (see Figure 4(a)), a good short-term forecast may not necessarily imply that the trend will persist. A thorough analysis with several different values of $K$ is needed to analyze other possible scenarios;
3) In a stationary situation where the past trends do not vary a lot, it is expected that the short-term trend will persist and will be consistent with the long-term future trend;

4) A good understanding of the timeline of the pandemic, along with the careful choice of $K$ and $n$ allows a methodical assessment of the impact of various intervention strategies and significant events surrounding the pandemic. This is demonstrated in Section IV where we presented a situational analysis of the third wave of the pandemic in Malaysia.

5) Our situational analysis also uncovers a plateauing phenomenon that is consistent with adaptive prevention strategies as shown in [51].

The extrap-KLMS algorithm is unique because, instead of just relying on point estimates of future values, the algorithm provide a spectrum of scenario-based predicted future trends and each future trends is specifically linked to a series of known past events. This offers the kind of flexibility policymakers need when making high stakes and possibly controversial decisions.

\section{REFERENCES}

[1] unsdg.un.org, "A UN framework for the immediate socio-economic response to COVID-19", Apr 2020. [Online]. Available: https://unsdg.un.org/resources/ un-framework-immediate-socio-economic-response-covid- 19. [Accessed: 17-02-2021]

[2] L. P. James, J. A. Salomon, C. O. Buckee and N. A. Menzies, "The Use and Misuse of Mathematical Modeling for Infectious Disease Policymaking: Lessons for the COVID-19 Pandemic", Medical Decision Making, vol. 582, 0272989X2199039, 2021.

[3] J. P.A. Ioannidis, S. Cripps, M. A. Tanner, "Forecasting for COVID-19 has failed", International Journal of Forecasting, 2020.

[4] I. Holmdahl and C. Buckee, "Wrong but useful - What Covid-19 epidemiology models can and cannot tell us", New Engl. J. Med., vol. 383, pp. 303-305, 2020.

[5] J. Tolles and T. Luong, Modeling epidemics with compartmental models. JAMA, 323(24), 2515-2516, 2020.

[6] W.O. Kermack and A.G. McKendrick, A contribution to the mathematical theory of epidemics. Proceedings of the Royal Society of London Series A:115(772), 700-721, 1927.

[7] M.H. Mohd and F. Sulayman, Unravelling the Myths of R0 in Controlling the Dynamics of COVID-19 Outbreak: a Modelling Perspective. Chaos, Solitons \& Fractals, 109943, 2020.

[8] A. Khan, R. Zarin, G. Hussain, N. A. Ahmad, M. H. Mohd, A. Yusuf, "Stability analysis and optimal control of covid-19 with convex incidence rate in Khyber Pakhtunkhawa (Pakistan)", Results in Physics, vol. 20, 103703, 2021, 103703.

[9] A. Kronbichler, D. Kresse, S.Yoon, K.H. Lee, M. Effenberger and J.I Shin, "Asymptomatic patients as a source of COVID-19 infections: a systematic review and meta-analysis", Int J of Inf Dis., vol. 98, pp. 180186, June 2020.

[10] M. Peirlinck, K. Linka, F. Sahli Costabal, E. Bendavid, J. Bhattacharya J.P.A. Ioannidis, E. Kuhl, "Visualizing the invisible: The effect of asymptomatic transmission on the outbreak dynamics of COVID-19", Computer Methods in Applied Mechanics and Engineering, vol. 372, 2020, 113410, https://doi.org/10.1016/j.cma.2020.113410.

[11] newscientist.com, 'Exclusive: Two variants have merged into heavily mutated coronavirus", Feb. 2021. [Online]. Available: https://www.newscientist.com/article/ 2268014-exclusive-two-variants-have-merged-into-heavily-mutated-coronavirus/. [Accessed: 17-02-2021]

[12] cdc.gov, "About Variants of the Virus that Causes COVID-19", Feb. 2021. [Online]. Available: https://www.cdc.gov/coronavirus/2019-ncov/ transmission/variant.html. [Accessed: 17-02-2021]

[13] who.int, "SARS-CoV-2 Variants", Dec. 2020. [Online]. Available: https://www.who.int/csr/don/31-december-2020-sars-cov2-variants/ en/. [Accessed: 17-02-2021] 
[14] R. Sujath, J.M. Chatterjee and A.E. Hassanien, "A machine learning forecasting model for COVID-19 pandemic in India", Stoch Environ Res Risk Assess, vol. 34, pp. 959-972 (2020). 10.1007/s00477-020-01827-8.

[15] F. Rustam et al., "COVID-19 Future Forecasting Using Supervised Machine Learning Models", IEEE Access, vol. 8, pp. 101489-101499, 2020, 10.1109/ACCESS.2020.2997311.

[16] Y. Mohamadou, A. Halidou and P.T. Kapen, "A review of mathematical modeling, artificial intelligence and datasets used in the study, prediction and management of COVID-19", Appl Intell, vol. 50, pp. 3913-3925, 2020. 10.1007/s10489-020-01770-9.

[17] T. Carletti, D. Fanelli and F. Piazza, "COVID-19: The unreasonable effectiveness of simple models", Chaos, Solitons \& Fractals: X, vol. 5, $100034(2020)$

[18] C. Sáez, N. Romero, J. A. Conejero and J. M. García-Gómez, "Potential limitations in COVID-19 machine learning due to data source variability: A case study in the nCov2019 dataset", Journal of the American Med ical Informatics Association, vol. 28, Issue 2, pp. 360-364, Feb. 2021, 10.1093/jamia/ocaa258.

[19] Y. Peng and M. H. Nagata, "An empirical overview of nonlinearity and overfitting in machine learning using COVID-19 data", Chaos, Solitons \& Fractals, vol. 139, Oct. 2020,110055, 10.1016/j.chaos.2020.110055.

[20] V. Chaurasia and S. Pal, S, "COVID-19 Pandemic: ARIMA and Regression Model-Based Worldwide Death Cases Predictions", SN COMPUT. SCI., vol. 1, no. 288, 2020. 10.1007/s42979-020-00298-6

[21] M. Saqib, "Forecasting COVID-19 outbreak progression using hybrid polynomial-Bayesian ridge regression model", Appl Intell, 2020. 10.1007/s10489-020-01942-7

[22] A. Ramchandani, C. Fan and A. Mostafavi, "DeepCOVIDNet: An Interpretable Deep Learning Model for Predictive Surveillance of COVID-19 Using Heterogeneous Features and Their Interactions", IEEE Access, vol. 8, pp. 159915-159930, 2020

[23] P. Wang, X. Zheng, G. Ai, D. Liu and B. Zhu, "Time series prediction for the epidemic trends of COVID-19 using the improved LSTM deep learning method: Case studies in Russia, Peru and Iran", Chaos, Solitons \& Fractals, vol. 140, 2020, 110214, 10.1016/j.chaos.2020.110214.

[24] N. Sheng, Q. Liu, S.J. Qin, T. Chai, IEEE Trans. Autom. Sci. Eng., vol. 13, No. 2, pp. 1129-1137, 7310889, Apr. 2016.

[25] J. Feng, J. Wang, H. Zhang, Z. Han, "Fault diagnosis method of joint fisher discriminant analysis based on the local and global manifold learning and its kernel version", IEEE Trans. Autom. Sci. Eng., vol. 13 , pp. $122-133,2016$

[26] C. Micchelli and M. Pontil, "Learning the kernel function via regularization”, Journal of Machine Learning Research, vol. 6, pp. 1099-1125, 2005.

[27] W. Liu, P. P. Pokharel and J. C. Principe, "The Kernel Least-MeanSquare Algorithm," IEEE Transactions on Signal Processing, vol. 56, no. 2, pp. 543-554, Feb. 2008, doi: 10.1109/TSP.2007.907881.

[28] J. Park, B. Lee, S. Kang, P. Y. Kim and H. J. Kim, "Online Learning Control of Hydraulic Excavators Based on Echo-State Networks," IEEE Trans. Autom. Sci. Eng., vol. 14, no. 1, pp. 249-259, Jan. 2017, doi: 10.1109/TASE.2016.2582213.

[29] E. Byon, Y. Choe and N. Yampikulsakul, "Adaptive Learning in TimeVariant Processes With Application to Wind Power Systems," IEEE Trans. Autom. Sci. Eng., vol. 13, no. 2, pp. 997-1007, April 2016, doi: 10.1109/TASE.2015.2440093.

[30] E. Kuhl, "Data-driven modeling of COVID-19-Lessons learned", $E x$ treme Mechanics Letters, vol. 40, 2020, 100921.

[31] K. Linka, M. Peirlinck, E. Kuhl, "The reproduction number of COVID19 and its correlation with public heath interventions", Comput. Mech. (2020) http://dx.doi.org/10.1007/s00466-020-01880-8.

[32] L. Magri and N.A.K. Doan, "First-principles machine learning modelling of COVID-19". Preprint, arXiv:2004.09478. 2020.

[33] F. Takens, "Detecting strange attractors in turbulence", Lecture Notes in Mathematics, vol. 898, pp. 366-381, 1981.

[34] H.D.I. Abarbanel, "Analysis of Observed Chaotic Data, Institute for Nonlinear Science", Springer-Verlag New York, 1996.

[35] E. Pelikán, "Tutorial:Forecasting of processes in complex systems for real-world problems", Neural Network World, vol. 24, pp. 567-589, 2014.

[36] S.L. Brunton and J.N. Kutz, "Data-Driven Science and Engineering: Machine Learning, Dynamical Systems, and Control", Cambridge, UK: Cambridge University Press, 2019.

[37] B. Scholkopf and A. J. Smola, "Learning with Kernels Support Vector Machines, Regularization, Optimization, and Beyond", Cambridge, MA, USA, USA: MIT Press, 2001.

[38] J.C. Principe, W. Liu and S. Haykin, "Kernel Adaptive Filtering: A Comprehensive Introduction”. 57. John Wiley \& Sons (2011).
[39] W. D. Parreira, J. Bermudez, M. Carlos, C. Richard and J-Y. Tourneret, "Stochastic Behavior Analysis of the Gaussian Kernel-Least-MeanSquare Algorithm", IEEE Transactions on Signal Processing, vol. 60, no. 5, pp. 2208-2222, 2012.

[40] B. Chen, S. Zhao, P. Zhu and J. C. Príncipe, "Mean square convergence analysis for kernel least mean square algorithm", Signal Processing, vol. 92, Issue 11, pp. 2624-2632, 2012, https://doi.org/10.1016/j.sigpro.2012.04.007.

[41] S.A. Lauer, K.H. Grantz, Q. Bi, F.K. Jones, Q. Zheng, H.R. Meredith HR, et al., "The Incubation Period of Coronavirus Disease 2019 (COVID19) From Publicly Reported Confirmed Cases: Estimation and Application", Ann Int Med., vol. 172, pp. 577-582, 2020.

[42] C. Del Rio and P. N. Malani, COVID-19-new insights on a rapidly changing epidemic. Jama, 323(14), 1339-1340, 2020.

[43] bbc.com, "Coronavirus: Italy imposes regional lockdown as Europe battles surges", Apr 2020. [Online]. Available: https://www.bbc.com/ news/world-europe-54839429. [Accessed: 20-02-2021]

[44] L. Rampal and B. S. Liew, Malaysia's third COVID-19 wave-a paradigm shift required. The Medical Journal of Malaysia, 76(1), pp. 1-4, 2021.

[45] T. Sukumaran, Coronavirus Malaysia: PM blames Sabah election as among causes of huge infection surge. The South China Morning Post. Available at: https://www.scmp.com/week-asia/healthenvironment/article/3104421/coronavirus-malaysia-pm-blames-sabahelection-among, 2020.

[46] R. Loheswar, Muhyiddin: Undocumented migrants cause of Sabah's Covid-19 situation, more detention centres to be opened. Malay Mail. Available at: https://www.malaymail.com/news/malaysia/2020/10/03/muhyiddinundocumented-migrants-cause-of-sabahs-covid-19-situation-more$\operatorname{det} / 1909146,2020$

[47] A. Wahab, The outbreak of Covid-19 in Malaysia: Pushing migrant workers at the margin. Social Sciences \& Humanities Open, 2(1), 100073 , 2020.

[48] K. Lappin, A Neoliberal Pandemic in Asia. International Union Rights, 27(4), 3-5,2020.

[49] A. Wahab, Migrant Workers and Covid-19 Outbreak in Malaysia (No. 3). Institute of Malaysian and International Studies (IKMAS), Universiti Kebangsaan Malaysia, 2020.

[50] M. Nik Anis, Travel restrictions lifted. The Star. Available at: https://www.thestar.com.my/news/nation/2020/12/09/travel-restrictionslifted, 2020.

[51] Z. Wu et al., COVID-19 Plateau: A Phenomenon of Epidemic Development under Adaptive Prevention Strategies. arXiv preprint arXiv:2011.03376, 2020.

Noor A. Ahmad received a Bachelor degree in Mathematics from the University of Bristol in 1993 and a Doctoral degree from the University of Southampton in 1997. She joined the School of Mathematical Sciences, Universiti Sains Malaysia in 1997 and became an associate professor in 2014 Her current research interests include machine learning, data-driven dynamical system, mathematical modelling and matrix methods in signal processing.

Mohd H. Mohd received his BSc (Hons) in Mathematics from Imperial College London, MSc in Mathematics from USM and PhD in Computational and Applied Mathematics from University of Canterbury, NZ. He is a researcher working in the fields of Mathematical Biology, Dynamical Systems and AgentBased Modelling. He has published numerous high-impact journals, peerreviewed international journals and also conference proceedings. He has also delivered various keynote, invited and contributed talks in many international conferences and seminars. Dr Hafiz is one of the Editors for Springer-Nature Edited Volume. He also serves as a reviewer of several international journals e.g., Chaos Solitons \& Fractals (Elsevier), IEEE Access (IEEE), Ecology and Evolution (Wiley), Theoretical Ecology (Springer), International Journal of Systems Science (Taylor \& Francis), Bulletin of the Malaysian Mathematical Sciences Society (Springer) and Environment, Development and Sustainability (Springer). 